\section{Electrochemical Capacitance of Mesoporous Gold}

\author{
M.B. Cortie, A.I. Maaroof and G.B. Smith \\ Institute for Nanoscale Technology, University of \\ Technology Sydney, PO Box 123, Broadway, NSW 2007, \\ Australia
}

\begin{abstract}
The surfaces of nanoscale gold particles and components are oxide-free under normal ambient conditions. This unusual attribute permits the exploration of microstructures and functionalities that would not be feasible for less noble metals. Here we consider the electrochemical properties of mesoporous gold sponges, prepared by de-alloying an $\mathrm{AuAl}_{2}$ precursor. The sponges have a high specific surface area, with an average pore diameter of $12 \mathrm{~nm}$, but are prone to sinter. They may be prepared in bulk, or, more usefully, as coatings. Their electrochemical capacitance divided by their nominal surface area is high and, at a cell voltage of $0.6 \mathrm{~V}$, reaches $100 \mathrm{mF} / \mathrm{cm}^{2}$ for bulk samples and $2 \mathrm{mF} / \mathrm{cm}^{2}$ for coatings. This is up to a thousand times greater than the 50 to $100 \mu \mathrm{F} / \mathrm{cm}^{2}$ exhibited by a planar gold surface.
\end{abstract}

\section{Introduction}

Mesoporous metallic surfaces may be prepared by dealloying suitable precursor intermetallic compounds. For example the Raney nickel and Raney copper catalysts may be prepared by de-alloying $\mathrm{NiAl}_{3}$ or $\mathrm{CuAl}_{2}$ respectively with a strong alkali [1,2], which dissolves out the aluminium to leave a nanoscale skeleton of noble metal. In general, however, the resulting materials readily oxidise and may even be pyrophoric. Gold is an exception to this rule, and stable, clean mesoporous surfaces and powders of it may be prepared [3]. The resulting powders and sponges have been shown to have catalytic activity [3,4] a point ostensibly in conflict [5] with the reigning paradigm $[6,7]$, which is that to be active as a catalyst gold must be present as discrete nano-sized particles bound to a transition metal oxide. However, we will not explore the catalytic properties of mesoporous gold further here save to note that it has been shown to be both a reasonable catalyst for the room temperature oxidation of carbon monoxide and for the reduction and oxidation of $\mathrm{NO}$ at 300 to $350^{\circ} \mathrm{C}$. In the present article we will rather address the electrochemical properties of this material and associated prospects for its exploitation.

Porous metal coatings have found actual or potential applications as electrodes in electrochemical devices such as ultra-capacitors [8] (also known as 'electrolytic double-layer capacitors' or 'super-capacitors') and in sensors of various kinds $[9,10])$. The use of these coatings in electrochemical devices is predicated on their very large specific surface area, which provides an increased surface upon which redox, ampometric and capacitive phenomena may occur. The enhanced surface area is the result of these materials containing a continuous network of nanoscale pores and channels. This effect is illustrated in Figure 1 which shows the total surface area (including occluded pores) of a mesoporous gold mass as function of the size and volume fraction of pores. This is a simplified geometry, and real samples do not have perfectly spherical pores, nor are the pores mono-disperse in size. Also, the simple calculation presented does not take into account issues of interconnectivity between the pores. Nevertheless, it suffices to illustrate the enormous increase in specific area that can be obtained in mesoporous metals, and how this surface area depends strongly on the size and volume fraction of the pores.

The idea of using gold nanoparticles for ultra-capacitance has already been mooted and prototyped by Raguse [11], and capacitances of the order of 2 to $3 \mathrm{~F} / \mathrm{g} \mathrm{Au}$ were demonstrated for a composite mass of alkane-dithiol coated nanoparticles. The charge in ultra-capacitors is stored as a double layer of charged ions on the surface of a conductor immersed in an electrolyte and values of 10 to $100 \mu \mathrm{F} / \mathrm{cm}^{2}$ are typical for clean gold [12] although this falls to 1 to 10 $\mu \mathrm{F} / \mathrm{cm}^{2}$ for thiol-coated surfaces. Therefore, the greater and cleaner the surface area, the greater the capacitance.

The existing processes for preparing porous or 


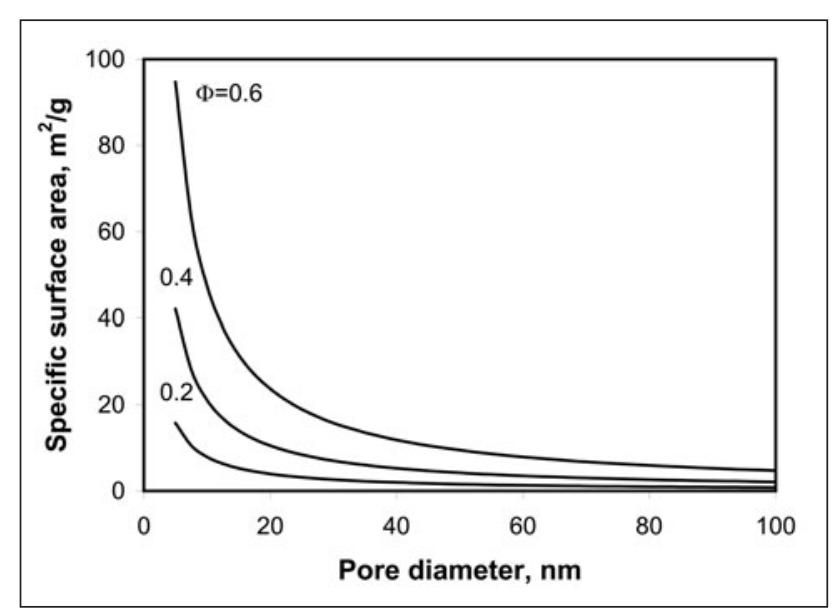

\section{Figure 1}

Specific surface area of mesoporous gold as a function of volume fraction of pores $(\Phi)$ and diameter of pores. This simplified calculation neglects issues of interconnectivity and pore shape. Note also that sponges with values of $\Phi$ greater than $\sim 0.5$ are very fragile and sinter readily. The best experimental value of specific surface area for this system is $20 \mathrm{~m}^{2} / \mathrm{g}[3,5]$

mesoporous metal films include powder metallurgy, which involves the sintering together of masses of fine metal powders, electrochemical reduction, which involves adjusting electrolytic plating conditions to produce a porous deposit rather than usual smooth deposit [13], chemical vapour deposition, deposition from a colloidal suspension and the dealloying of intermetallic precursors mentioned previously. However, the existing methods do not provide for all desired configurations of mesoporous metals and there is a great convenience to be had from creating the films from a vacuum-deposited coating. Curiously, while the ideas of dealloying $\mathrm{AuAl}_{2}$ to produce mesoporous gold powder [3], and of applying films of $\mathrm{AuAl}_{2}$ by vacuum deposition for decorative [14] or tribological [15] applications are known, there appears to have been no recognition, at least in the literature, that the ideas could be combined into one to produce mesoporous gold films. Therefore, we have developed this method to provide the desired properties of very high surface area while keeping the film thickness exceedingly thin. This provides, for the first time, a means to achieve a large surface area of mesoporous gold at an economic cost.

\section{Experimental}

Samples of mesoporous gold were prepared by the controlled de-alloying of the intermetallic compound AuAlz. This intermetallic precursor material is commonly known as 'purple gold' [16] or 'purple glory' [17] on account of its attractive purple colour. Two forms of the precursor material were compared in this work, namely solid buttons and vacuum-deposited thin films. The buttons were produced in a vacuum-arc furnace, and contained $2.0 \mathrm{~g}$ of gold, to make a total mass of about $2.5 \mathrm{~g}$, depending on Al content. A small loss of $\mathrm{Al}$ of the order of $0.01 \mathrm{~g}$ occurred on melting. The thin films of $\mathrm{Au}_{\mathrm{x}} \mathrm{Al}_{\mathrm{y}}$ were produced by simultaneously sputtering $\mathrm{Au}$ and $\mathrm{Al}$ from two elemental targets of $50 \mathrm{~mm}$ diameter using a high vacuum DC magnetron sputtering apparatus. The rates of sputtering were controlled by varying the power delivered, and the system calibrated to produce a stoichiometry close to AuAlz. The quantity of gold deposited was equivalent to $\sim 20 \mathrm{~nm}$ thickness if deposited alone. The amount of Al deposited was equivalent to a pure coating of $\sim 160 \mathrm{~nm}$ thickness. These quantities were determined using the relative densities of the elements and the desired stoichiometry of the target compound. Glass slides were used for the substrate and they were heated to $400^{\circ} \mathrm{C}$ during co-deposition using an in situ heater. However, while the AuAlz precursor coating adhered readily, adhesion of the subsequent mesoporous gold was poor unless the glass surface had been pre-treated first by depositing a $100 \mathrm{~nm}$ thick layer of chromium metal. De-alloying was carried out using $4 \mathrm{M} \mathrm{NaOH}$ at room temperature in the case of the bulk samples, and $0.044 \mathrm{M} \mathrm{KOH}$ in the case of the coated samples.

The discharge capacitance of the electrodes was measured in a system which comprised a constant voltage power supply, an A/D converter, and a stirrer-hotplate. The electrolyte for the buttons was $4 \mathrm{M} \mathrm{NaOH}$, whereas for the coated samples it was $0.1 \mathrm{M} \mathrm{Na}_{2} \mathrm{SO}_{4}$, or $0.1 \mathrm{M}$ $\mathrm{Na}_{2} \mathrm{SO}_{4}+0.044 \mathrm{M} \mathrm{KOH}$. The circuit is shown in Figure 2. Cyclic voltammetry (CV) was also performed on a gold electrode in $0.1 \mathrm{M} \mathrm{Na}_{2} \mathrm{SO}_{4}$ to provide information on the oxidation, reduction and double layer capacitance of the Au electrodes under standard conditions. In this case a gold electrode of 1 $\mathrm{mm}$ diameter was first cleaned by 10 cathodic cycles, followed by 10 anodic cycles, after which it appeared to exhibit a stable CV characteristic when cycled between -0.3 $\vee$ to +1.7 V vs SCE. (Add $0.22 \mathrm{~V}$ to get the potential vs SHE.) This range encompasses the known region of simple double layer capacitance in gold [8].

The ostensible capacitance of the system was determined from discharge experiments using the equation

$$
V(t)=V_{0} \exp (-t / R C)
$$

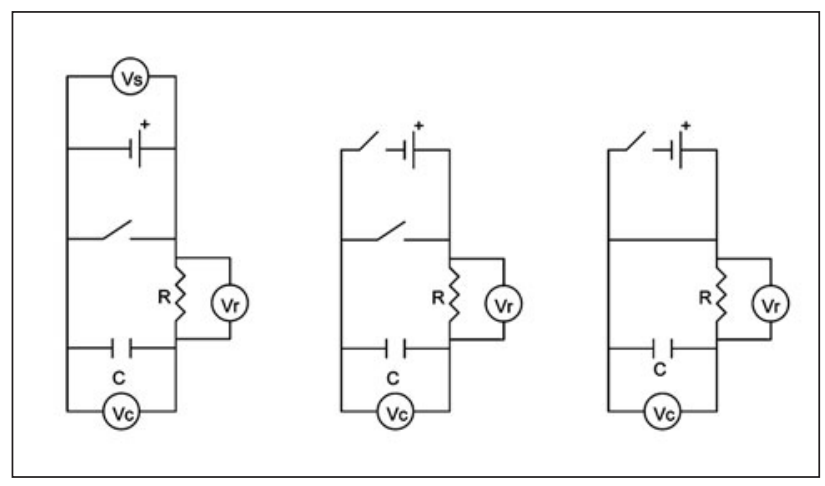

Figure 2

(a) Charging the capacitor, $V_{c}+V_{r}=V_{s}, V_{s}=$ constant, $V_{c} \neq V_{r}, V_{c}, V_{l}=f(t)$, (b) capacitor on open circuit, $V_{c}=0, V_{c}=$ constant, (c) discharging capacitor, $V_{c}=V_{r}=f(t)$. $R$ equals $8.2 K$ in this work 
and in the case of cyclic voltammetry from the relation

$$
C=i / s
$$

where $i$ is the current and $S$ the scan rate.

In both cases $C$ may also be given by the entirely equivalent expression

$$
C=\frac{d Q}{d V}
$$

The term 'ostensible capacitance' has been introduced here to encompass all the capacitance in the system, including non-Faradaic double layer capacitance, any possible Faradaic pseudo-capacitance of the type introduced by Conway [8], and supply of electrical energy from any irreversible, batterylike process.

\section{Results}

\subsection{Microstructure}

Both as-cast buttons and evaporated coatings exhibited the bright purple colour of $\mathrm{AuAl}_{2}$, confirming that this was the majority phase in both instances. Significant hydrogen
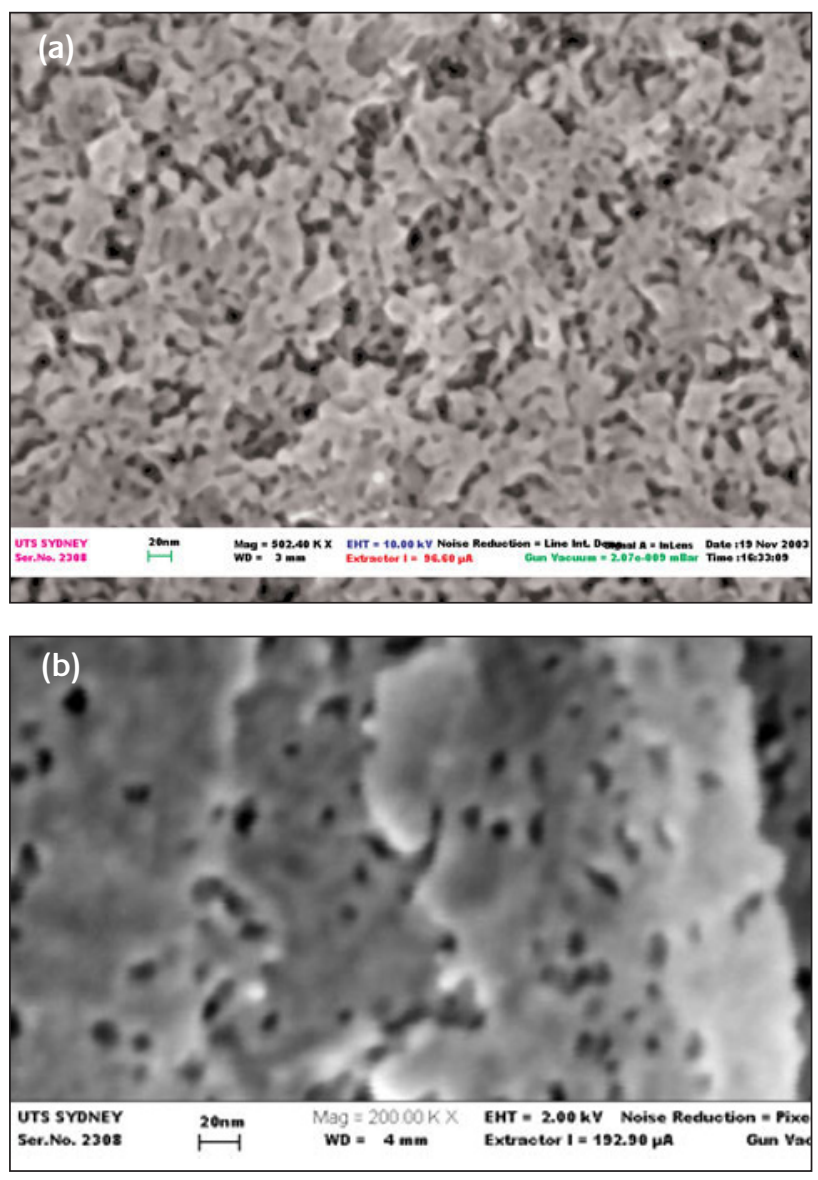

Figure 3

Surface morphology of mesoporous gold, (a) thin coating of mesoporous gold on glass, showing pores and channels of between 5 and $20 \mathrm{~nm}$ diameter, (b) bulk mesoporous gold sample, showing a sparse distribution of pores of 10 to $15 \mathrm{~nm}$ diameter on a surface of re-coalesced gold sponge evolution occurred when samples were leached, and the colour of the surfaces changed to black. The reaction seemed complete in the case of the coatings in less than 5 seconds. However, the buttons continued to de-alloy over several weeks, with periods of passivation followed by intermittent activity.

The surface of the thin films when freshly produced was very porous, Figure 3(a), and it can be seen that there are a multiplicity of interconnected channels. The surface of the button samples was less porous, Figure 3(b). In both cases it is quite evident that the surfaces were partially sealed by an inert layer of gold early on in the de-alloying process. This layer blocked the egress of hydrogen generated deeper down in the material, and the resulting build-up of pressure caused a multiplicity of 'bursts' on the de-alloyed surface, even on the thin coating. This phenomenon was observed on the surfaces of both coated and bulk samples. The surfaces are very soft, as expected.

It was observed that the porosity was dependent upon the etching conditions, and that considerable coalescence and shrinkage of the mass of mesoporous gold occurred unless the temperature during etching was kept low. This sintering might seem odd, given that the samples were at no time hotter than $50^{\circ} \mathrm{C}$, but it should be borne in mind that not only is the melting point of nano-structured gold substantially depressed [18,19], but also that the gold atoms left behind on the surface after de-alloying are not laterally coordinated, and will tend to migrate to and condense onto clusters of their kind [20].

A specific surface area of $20 \mathrm{~m}^{2} / \mathrm{g}$, as measured by BET and $\mathrm{N}_{2}$, is achievable under optimum conditions $[3,21]$. This surface area can be shown to be geometrically equivalent to that of a loose agglomeration of gold particles of $7 \mathrm{~nm}$ diameter. Although the surface area falls far short of the $1000 \mathrm{~m}^{2} / \mathrm{g}$ or so of activated carbon, the comparison is more favourable once the density of the two elements has been taken into account. Mesoporous gold has a surface area per unit volume that is about a third of the value achievable with carbon. The pore sizes produced in three types of mesoporous gold were measured by analysis of high resolution SEM images with the assistance of a computer program that superimposed a circle of approximately matching diameter over the pore opening. The results are shown in Figure 4. It is evident that pore diameters are in the range 6 to $18 \mathrm{~nm}$, with a preponderance at around $12 \mathrm{~nm}$.

\subsection{Cyclic voltammetry of gold}

The electrochemical behaviour of gold electrodes has been well studied, particularly in respect of the formation and reduction of surface oxides, and the associated reduction of oxygen [e.g. 22-25]. In the course of this work it has been found that a prior cathodic treatment accelerated subsequent oxide formation during an anodic excursion, with the effect exacerbated for prolonged prior cathodisation [24]. In the present work the gold electrode was first cycled ten times between -1.5 and $0 \mathrm{~V}$ vs SCE to clean the surface. 

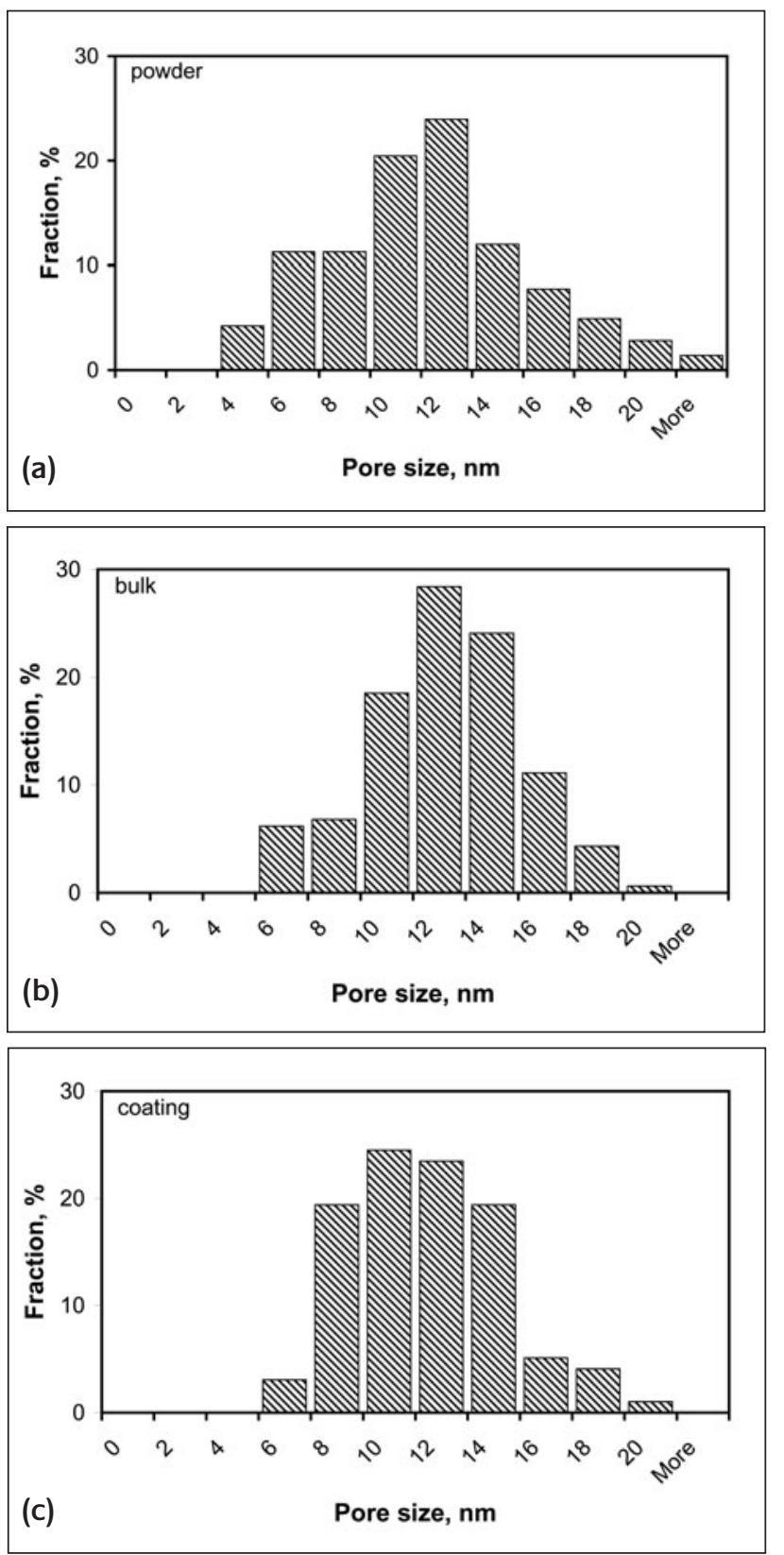

\section{Figure 4}

Pore size distributions, (a) mesoporous catalyst powder, manufactured by Mintek, South Africa, (b) bulk samples, (c) coatings on glass slides

Following this, the electrode was anodically cycled between 0 and +1.5 V vs SCE until a stable signal was established, thereby permitting determination of the double layer capacitance (Figure 5). The capacitance during the forward scan varies as a function of voltage from zero to about 100 $\mu \mathrm{F} / \mathrm{cm}^{2}$, which is consistent for previously reported values for this system. However, during the reverse scan the capacitance starts off noticeably lower. This may be because the surface of the gold was then coated with some oxide. Also evident in Figure 5 is the fact that the capacitance of a bare gold surface and of the presumably oxidised surface fall to zero at $-0.08 \mathrm{~V}$ and $+1.05 \mathrm{~V}$ vs SCE respectively. In the former case this corresponds closely to the known potential of zero charge (PZC) for bare gold, which is in the range -0.08 to -0.12 V vs SCE [26]. It is possible therefore that the

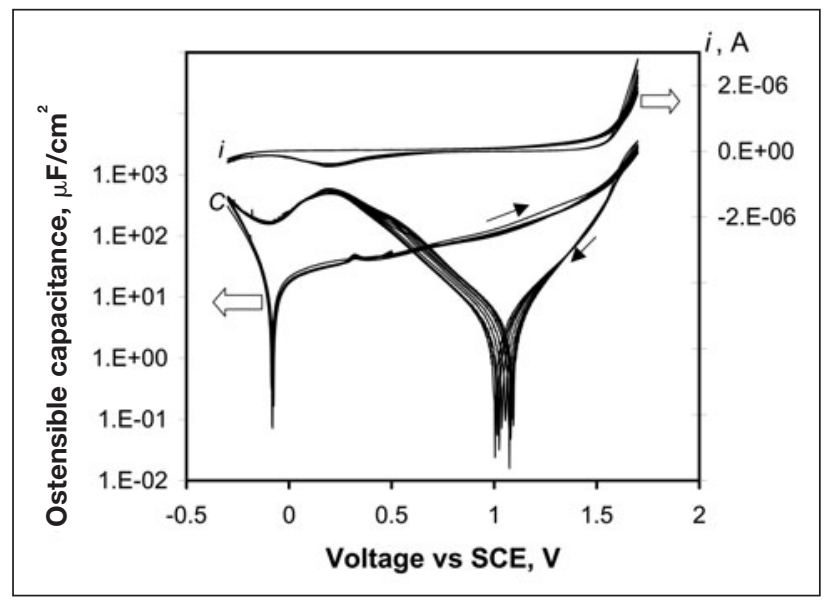

\section{Figure 5}

Cyclic voltagram (upper curve) and ostensible capacitance of gold electrode (lower curve, logarithmic scale) when cycled between -0.3 V to $+1.7 \mathrm{~V}$ vs SCE in $0.1 \mathrm{M} \mathrm{Na}_{2} \mathrm{SO}_{4}$

minima in both forward and reverse scans are related to the PZC's of the respective surfaces.

The oxidation process occurring on the surface of the gold is complex, and in this case is likely to involve the simultaneous and competitive adsorption of the $\mathrm{SO}_{4}{ }^{2-}$ anion onto the surface, and its possible incorporation into the oxide [23]. Oxidation in this system begins at about $+1.1 \mathrm{~V}$ vs SCE, and is accentuated by carrying the scans deep into the anodic region, Figure 6 . However, there is considerable hysteresis in the process, and the reduction of the oxide during the reverse scan occurs at a rather more negative potential. It is known that the greater the extent of oxide formation or the higher the potential at which it was formed, then the lower the potential at which it is reduced [23]. The reduction peak can be moved more than $0.5 \mathrm{~V}$ by this mechanism [23]. For an ideal pseudo-capacitive system, such as occurs on ruthenium, the reaction should have been reversible in an electrochemical sense, with the potentials of oxidation and reduction being approximately equal [8].

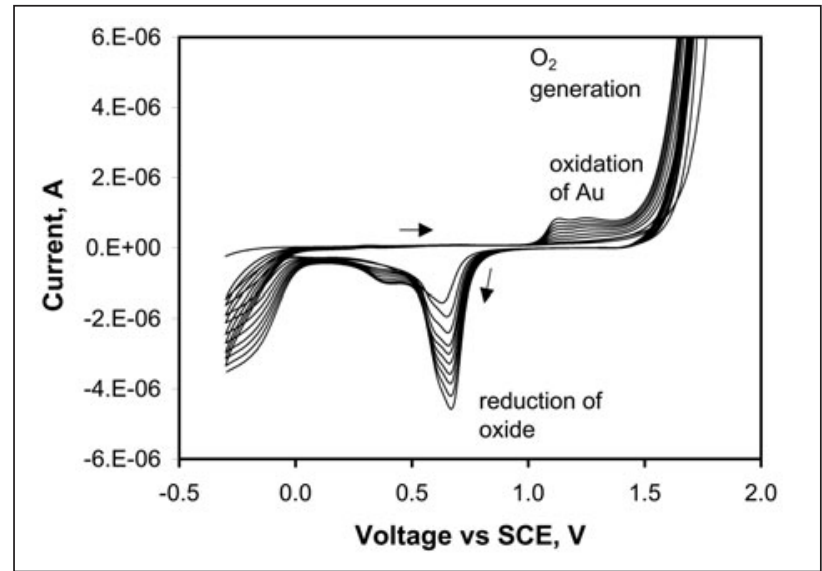

\section{Figure 6}

Cyclic voltagrams of $1 \mathrm{~mm}$ diameter gold electrode in $0.1 \mathrm{M} \mathrm{Na}_{2} \mathrm{SO}_{4}$ solution showing increasing intensity of oxide formation and reduction when electrode is cycled deep into oxidising region, to $+2.0 \mathrm{~V}$ vs SCE 
However in the scans shown in Figure 6 the potential range was fixed, and the net effect of cycling has been only to increase the surface area of material involved in each oxidation-reduction cycle. This is shown by the increasing amplitude of the reduction peak. The process of repeated oxidation followed by reduction is known to induce considerable roughening of the gold surface [27], which can readily explain the increase in surface area and capacitance achieved here. The nature of the cathodic reaction which started and ended at $0 \mathrm{~V}$ vs SCE is puzzling, but it may be related to localised $\mathrm{pH}$ changes around the electrodes in this unbuffered electrolyte.

\subsection{Electrochemical properties of de-alloyed buttons}

The buttons were inserted into the circuit shown in Figure 2, and charged with either an anodic or a cathodic potential, followed by periods of discharge over the resistor. The imposition of an anodic potential on the buttons appeared to accelerate the de-alloying reaction, but even when this was continued for several days, residual Al was still present in an electrochemical sense. Evidence for this was that the buttons exhibited an intermittent tendency to polarize to $-0.8 \mathrm{~V}$ relative to the inert counter electrode. This is the voltage that a freshly exposed surface of AuAlz manifests relative to an inert counter electrode in a corrosive alkaline solution.

Even when no active corrosion events occurred, the discharge curves of electrodes polarised in either the cathodic or anodic directions turned out to be a complex function of prior history. As a general rule, severe prior cathodic or anodic polarisation caused a slower discharge, i.e. higher ostensible capacitance. However, other factors such as time at charge and maximum cell voltage also played as yet unresolved roles. The complicating influence of prior treatment on the electrochemical behaviour of gold surfaces is well known [e.g. 25]. A range of cathodic and anodic discharge curves have been plotted in Figures 7 and 8 respectively. It is clear that there are one or more processes, in addition to double layer capacitance, that delay the discharge of the electrodes. In the case of the cathodic curves one of these may be the dissolution of residual Al, which, if it occurs, can hold the voltage for a while at $-0.8 \mathrm{~V}$ vs the inert counter electrode. However, this is not a possible explanation the anodic discharge curves shown here, which are characterised by some power-producing process that switches on at between about +1.0 and $+0.7 \mathrm{~V}$ vs the counter electrode during a discharge. The onset of this process can be sufficiently intense as to cause a temporary rise in the cell voltage, as in curves a and b in Figure 8. We will return later to this point and will show how the process producing the electric power might be driven by the metastability of freshly produced mesoporous gold surfaces. The ostensible capacitances associated with the anodic discharge curves are shown in Figure 9, and are of the same order as those determined from the cathodic discharge curves. In general, the capacitance exhibited by these electrodes is in the range

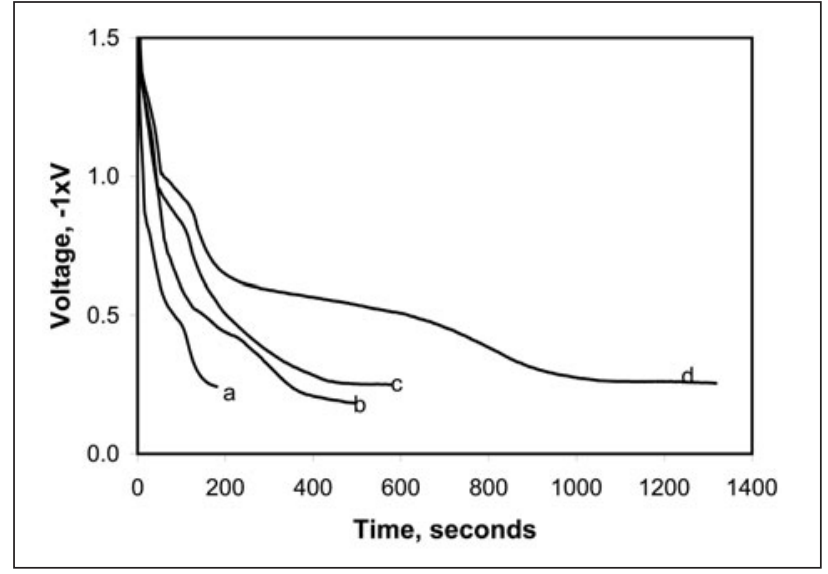

Figure 7

Cathodic discharge curves of mesoporous gold buttons polarised to $-1.8 \mathrm{~V}$ vs inert counter electrode. Electrolyte is $4 \mathrm{M} \mathrm{NaOH}$, (a) after 27 seconds of charging, (b) after 77 seconds of charging, (c) after 353 seconds of charging, (d) after 2380 seconds of charging

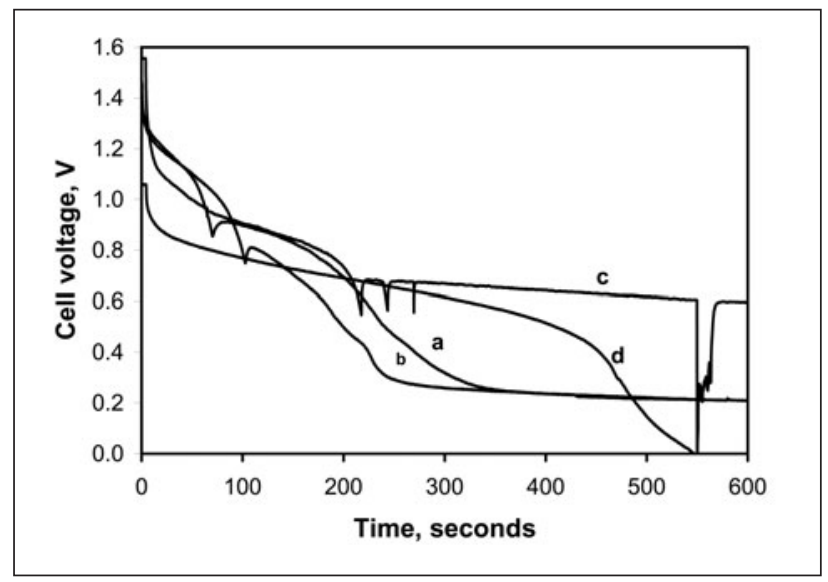

\section{Figure 8}

Anodic discharge curves in $4 \mathrm{M} \mathrm{NaOH}$ showing the simultaneous effects of an electrochemical process between +0.6 and +0.9 V that holds up the voltage, and the intermittent dissolution of $\mathrm{Al}$, which sporadically polarizes the electrode to $-0.8 \mathrm{~V}$, (a) after two days of cathodic charging and discharging, (b) discharge immediately following a, (c) after 720 minutes of anodic polarization and one cathodic cycle, (d) after 2700 minutes of anodic polarization

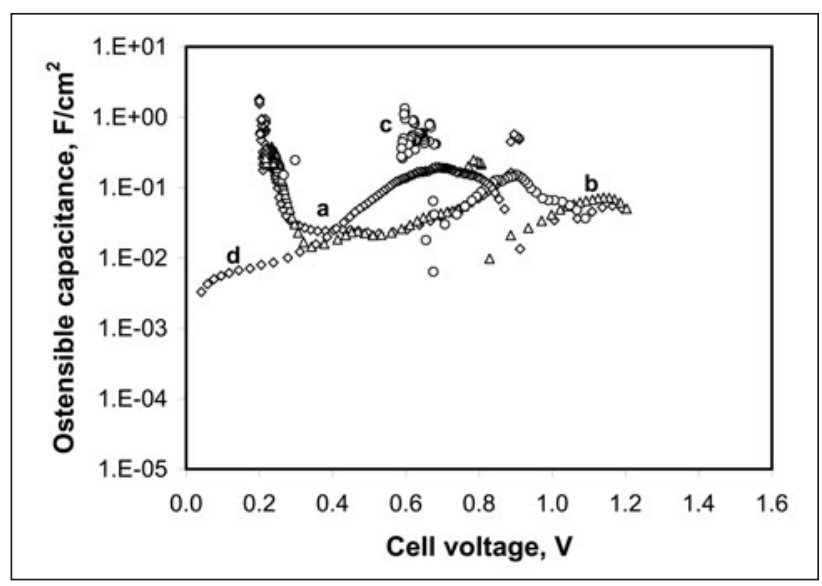

Figure 9

Ostensible capacitance exhibited by the anodic discharge curves shown in Figure 8 


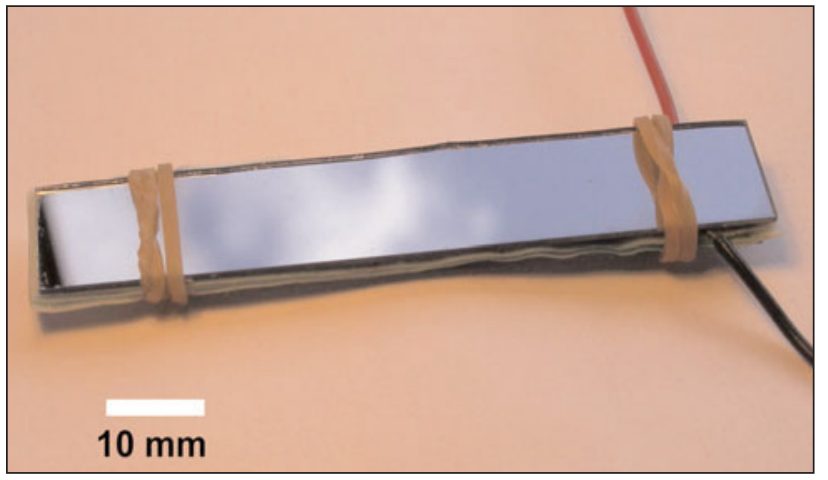

Figure 10

Prototype ultra-capacitor made by evaporating AuAlz onto glass slides, followed by making a sandwich of the glass slides with a filter paper spacer

of $1 \times 10^{-2} \mathrm{~F} / \mathrm{cm}^{2}$ to $1 \times 10^{-1} \mathrm{~F} / \mathrm{cm}^{2}$. These are very high values, and are the result of a very large surface area of sponge in the button. However, since the buttons contain $2 \mathrm{~g}$ of gold, the values represent only $5 \times 10^{-3}$ to $5 \times 10^{-2} \mathrm{~F} / \mathrm{g} \mathrm{Au}$, which is an inefficient use of the precious metal.

\subsection{Prototype ultracapacitors made from $\mathrm{AuAl}_{2}$ coatings}

Figure 10 shows a prototype electrochemical capacitor prepared from two glass slides, the inner surfaces of which were coated with mesoporous gold. The coated surfaces were kept apart by a water-permeable barrier of filter paper, while current leads were attached as shown. The assembly was held together by rubber bands, and charged and discharged over a resistor of $8.2 \mathrm{k} \Omega$ using a constant voltage supply.

Discharge curves and ostensible capacitance for the prototype device are compared in Figure 11 to data measured for a reference device made the same way, but with ordinary gold coatings. The electrolyte in both cases was $0.1 \mathrm{M} \mathrm{Na}_{2} \mathrm{SO}_{4}$. In this comparison the polarity of the charge cycles was alternated in an attempt to develop a symmetrical surface structure on both electrodes. It is evident that this treatment produced a relatively symmetrical discharge behaviour for the electrodes coated with ordinary gold, but failed to for the device made from electrodes coated with mesoporous gold. The capacitance developed is shown for the technologically interesting cell voltages between 0.6 and $1.3 \mathrm{~V}$, and in one of the bias directions of the mesoporous device it was up to six times greater than for ordinary gold, while for the other bias direction it was of the same order. The increase in ostensible capacitance as a function of cycling is quite pronounced.

The data in these figures were measured for freshly made samples. In fact repeated cycling of all kinds of gold-based electrodes increases their charge storage capacity significantly. This appears to be due to the development of a porous surface in the case of the ordinary gold surfaces, or to the further development of surface roughness in the case of the devices initially made from mesoporous coatings.
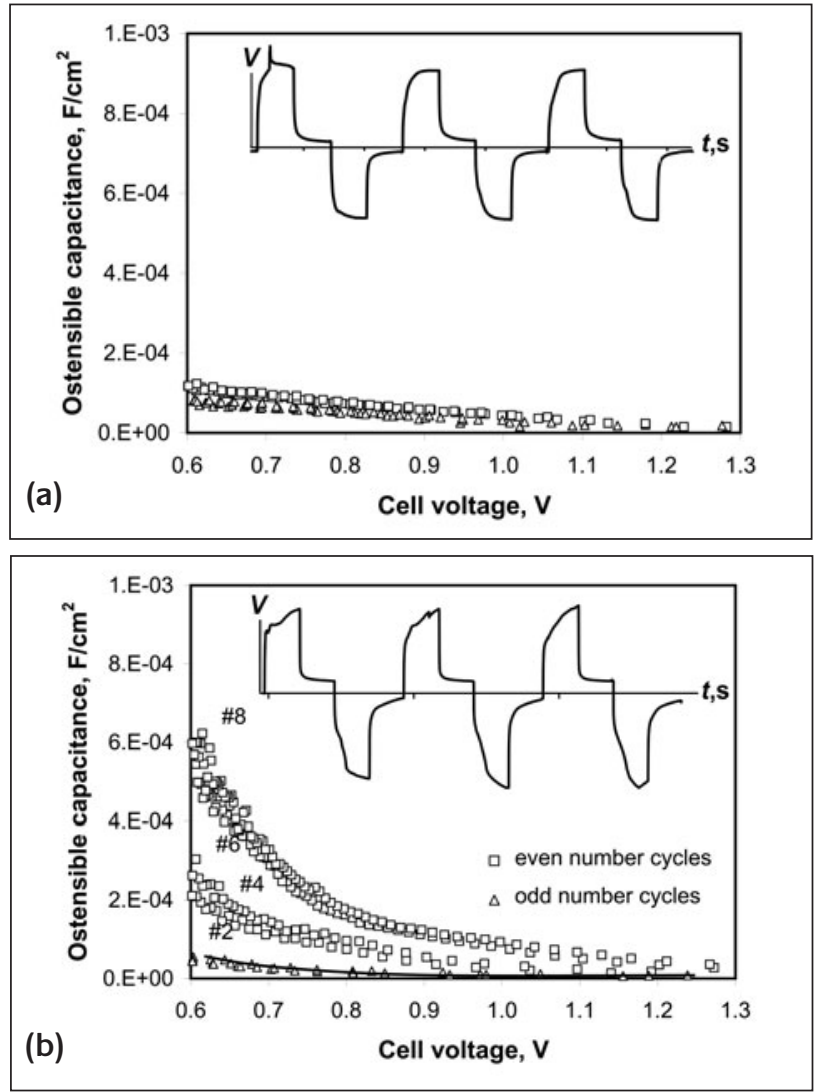

\section{Figure 11}

Ostensible capacitance of experimental ultracapacitors made using (a) pure gold coatings and (b) coatings of mesoporous gold. In the latter case it can be seen the there was a pronounced enhancement in capacitance during the even numbered cycles, which are the ones plotted for $\mathrm{V}<0$ in the inset. The electrolyte is $0.1 \mathrm{M} \mathrm{Na}_{2} \mathrm{SO}_{4}$

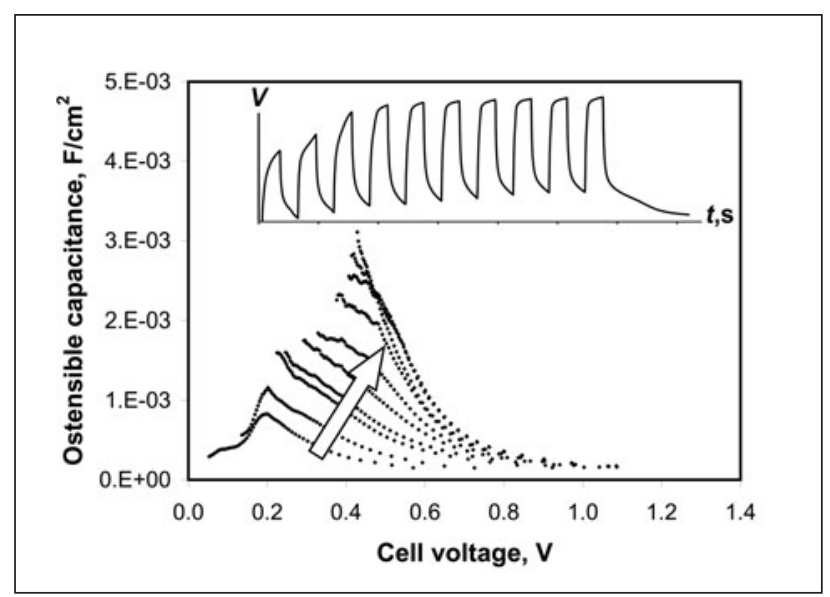

Figure 12

The increase in ostensible capacitance as a function of cell voltage (main graph) as the result of the application of ten consecutive charge/discharge cycles of 1 minute duration each (inset)

A sequence comprised of consecutive 1 minute charge and discharge cycles and its associated capacitance is shown in Figure 12. It is evident that the capacitor did not discharge completely in one minute, and that its capacitance systematically increased during the sequence. At $0.5 \mathrm{~V}$ the capacitance rose eightfold, from $2.3 \times 10^{-4} \mathrm{~F} / \mathrm{cm}^{2}$ for the first discharge cycle to $1.97 \times 10^{-3} \mathrm{~F} / \mathrm{cm}^{2}$ for the last. 


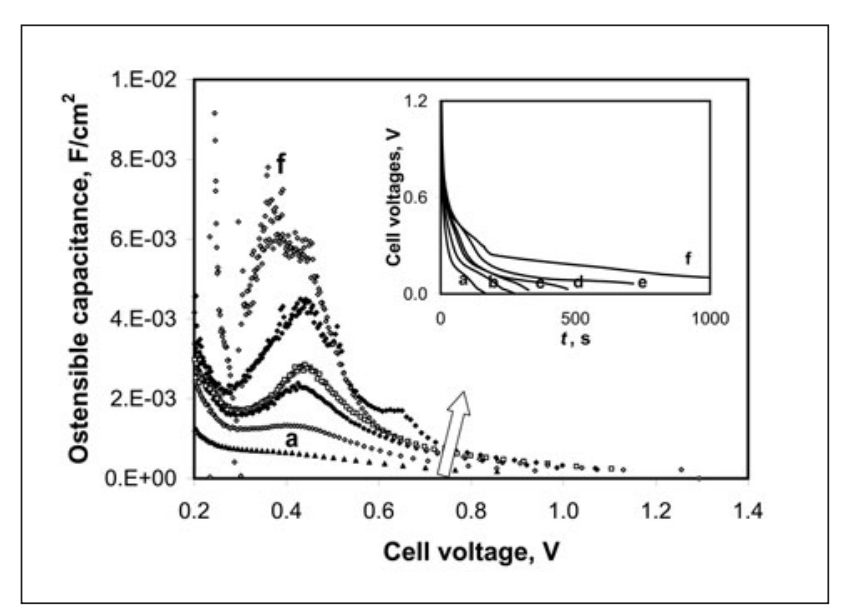

\section{Figure 13}

Ostensible capacitance as a function of cell voltage for the discharge curves (inset) obtained after charging times of (a) 30 seconds, (b) 1 minute, (c) 2 minutes, (d) 4 minutes, (e) 8 minutes and (f) 16 minutes. The pronounced increase in capacitance as a function of cycle and/or charging time is evident

As mentioned previously, the amount of charge stored is a function not only of repeated cycling, but also of how long the cell voltage was maintained on charge. This is illustrated in Figure 13, which shows the ostensible capacitances and discharge voltages recorded for a series of charge cycles of increasing duration.

\section{Discussion}

\subsection{Electrochemistry of gold ultracapacitors}

The electrochemistry of gold surfaces has been much studied [e.g. 23,24,25]. In broad summary the formation of gold oxide starts at about +1.1 to $1.2 \mathrm{~V}$ vs SCE on an annealed sample, but may start at potentials of as low as $+0.2 \mathrm{~V}$ vs SCE on a severely cathodised sample [25]. The higher of these two potentials corresponds to the formation of an $\alpha$ oxide, which has a relatively dense and ordered oxide $\left(\mathrm{Au}_{2} \mathrm{O}_{3}\right)$, while the less dense and more disordered hydrous $\beta$ oxide of gold $\left(\mathrm{Au}(\mathrm{OH})_{3}\right)$ is generated (and reduced) at the lower potential [25]. Oxygen evolution will occur at potentials of from $1.6 \mathrm{~V}$ vs SCE upwards. The redox behaviour of a gold surface is further complicated by the fact that severe anodisation may convert the $\alpha$ oxide to hydrous $\beta$ oxide [24].

In addition, however, gold atoms are quite mobile at the nanoscale, a phenomenon which manifests as, for example, a significant lowering of the melting point of gold nanoparticles $[18,19,21]$. The very rough, nanoscale structures produced by de-alloying are also effected by this phenomenon [20]. Therefore, the charge stored in the double layer on the surface may become a function of time, since [28]

$$
Q=C \cdot A(t) \cdot\left(E_{2}-E\right)
$$

where $C$ is capacitance per unit area, $A(t)$ the area as a function of time, $E$ the voltage, and $E_{z}$ the potential of zero charge. So, all else being equal, if $A$ changes, a current will flow, even if $\mathrm{d} E / \mathrm{d} t=0$ [28]. As mentioned, when a gold electrode that has been oxidised during an anodic charge cycle is subsequently reduced, the surface immediately resulting from this action is generally very rough and porous $[23,25,27,28]$. The effect is exacerbated if carried out on the already porous surface of mesoporous gold. However this new surface area subsequently re-coalesces due to so-called electrochemical annealing [23] and a current must flow for the reasons given above. It may be this current (with its associated potential) that appears to prolong the power generated during a discharge after anodic charging.

Although the increase in capacitance of the present electrodes may be induced by either prolonged holding times (as in Figure 14), or by cycling (Figure 13), it seems that in general cycling of potential is expected to be the more effective strategy for securing an increase in surface roughness [23].

\subsection{Possible applications of mesoporous gold capacitance}

Here we will briefly discuss the prospects of using mesoporous gold surfaces to make 'ultracapacitors' or electrochemical biosensors.

Ultra-capacitors draw their competitive advantage from the huge surface area of the conductor, and the very small distance, $d$, between the oppositely charged layers of adsorbed ions. This is because capacitance, $C$, is proportional to the surface area of conductor and inversely proportional to $d$. The stored energy $U_{e}$, is however

$$
U_{e}=\frac{C \cdot \Delta V^{2}}{2}
$$

where $V$ is the voltage across the plates. For example, a 10 g parallel plate capacitor might have $A$ and $d$ equal to 0.5 $\mathrm{m}^{2}$ and $10 \mu \mathrm{m}$ respectively, which, coupled with an $\epsilon_{\mathrm{r}}$ of 4 , yields a capacitance of the order of $2 \mu \mathrm{F}$, corresponding to about $6 \mu \mathrm{J}$ of stored energy (at 2.5V). In comparison, a similar-sized ultra-capacitor based on carbon-powder may have an effective $A$ of $5000 \mathrm{~m}^{2}$ and a $d$ of $2 \mathrm{~nm}$ [29], to yield a capacitance of about $80 \mathrm{~F}$ and a stored energy content of $250 \mathrm{~J}$, forty million times greater! Ultracapacitors have considerably better power buffering capacities than chemical batteries or ordinary capacitors [29-31]. This follows because power is the rate of energy transferred per unit time and, although ultra-capacitors cannot match the energy storage density of chemical batteries, they can charge and discharge much faster. This gives ultra-capacitors a special niche in electric circuits [30].

The possible use of mesoporous gold in ultra-capacitor technology is predicated upon the high electrical conductivity of gold, relative to for example, carbon powder. However, ultra-capacitors based on carbon powder obviously start off with a significant economic advantage. Nevertheless, mesoporous gold might have some 
applications in this area because carbon-based ultracapacitors suffer limitations in performance at high power ratings due to the internal resistance of their porous carbon aggregates, which limits power draw [31]. After accounting for energy lost to internal heating, the peak power that can be drawn from an ultra-capacitor while discharging it from voltage $V_{0}$ to $1 / 2 V_{0}$ is given by [30]:

$$
P=\frac{9}{16}(1-E f) \frac{V_{0}^{2}}{R}
$$

where $P$ is the power drawn from the ultra-capacitor, $E_{f}$ is the discharge efficiency (i.e. if $E_{f}=0.8$ then 0.2 or $20 \%$ of the energy is dissipated as heat), and $R$ is the internal resistance. The upshot of this is that the power that can be supplied by an ultra-capacitor is inversely proportional to the internal resistance. Therefore, halving this latter figure will double the power. It will be noted that the power is also sensitive to the maximum voltage, but this is strictly limited by the breakdown potential of the electrolyte used, being about $2.5 \mathrm{~V}$ for organic electrolytes and $1 \mathrm{~V}$ for aqueous electrolytes, and little further improvement seems feasible from this source [30]. However, it is with respect to the reduction of $R$ that gold sponge might offer some advantage.

However, any ultracapacitor produced from gold sponge will exhibit an electrical performance that is controlled in a very complex way by its prior history of charging and discharging. It has been shown here that such a device would exhibit a minimum capacity to store charge after a lengthy period of disuse, but would then increase in capacity with subsequent cycles of charge and discharge up to some limiting magnitude. This attribute would need to be taken into account in any attempt to make practical use of this system for energy storage. Furthermore, although the conductivity of the gold is immensely superior to that of carbon particles, there is still the issue of the conductivity of the electrolyte itself, which will cause its own inefficiencies due to Equation (6).

The capacitance offered by a mesoporous gold surface may also be potentially exploited for applications in sensors. Any conceptual biosensor [e.g. 32] that is based on a capacitive signal on a gold substrate can benefit from the increased capacitance offered by the high specific area of mesoporous coatings. In such a sensor, the surface is used as an electrically-conductive, porous scaffold on which to assemble functionalized coatings of an attached molecule that has a selective affinity for a target analyte. Gold surfaces are a very appropriate basis for such 'self-assembly' due to their metallic nature, great nobility, and particular affinity for sulphur. This allows functionalization with thiols of various types as well as direct adhesion to diverse biological molecules once they have been modified to contain a sulphur atom [33]. Binding of the analyte to the sensor will cause a change in capacitance which may be detected using simple instrumentation based around a voltage source and a means to measure the cell voltage as a function of time. If the molecule bound to the surface is, for example, an antibody to some disease organism, then the presence of the disease organism will be indicated.

Alternatively, it seems possible that the known catalytic efficacy of mesoporous gold could be directly exploited to detect an analyte by a selective chemical reaction, such as for example oxidation. There are a number of wellestablished techniques for chemical analysis that require the electro-oxidation of an analyte on the surface of a gold electrode. The sensitivity of such a technique depends in general on the product of the faradaic current density generated by the redox reaction of interest and the surface area of the electrode. Ostensibly, one way to increase sensitivity would be to use an electrode of greater than nominal geometric surface area by increasing its surface roughness [34]. However, the possible tortuous morphology of any pores can restrict mass transport of ions and reactants and there may not actually be any enhancement in the faradaic (redox) current [34]. However, the huge increase in the ratio of capacitive to faradaic current possible in mesoporous systems implies that any electrochemical sensor that is primarily based on the properties of the electrochemical double layer should benefit from being made of mesoporous gold.

Mesoporous gold can also potentially serve as a superior in vivo electrode, since it can both conduct electricity and serve as a storage reservoir for biologically-active molecules. The efforts being made to promote 'porous silicon' in this context are interesting, and it is possible that a gold-based device might display competitive properties. The controlled release of model pharmaceutical compounds from porous deposits of electroless nickel has recently been demonstrated [35], suggesting that the idea may be practicable.

\section{Conclusions}

Highly porous gold surfaces may be prepared by de-alloying an $\mathrm{AuAl}_{2}$ precursor prepared by melting and casting or by vacuum co-deposition. The resulting material is mesoporous, with pore diameters of the order of $12 \mathrm{~nm}$. It displays a much increased electrochemical double layer capacitance for this reason, and this effect has been demonstrated here using both single and double electrode configurations. At a cell voltage of $1 \mathrm{~V}$, ostensible capacitances of up to $10^{-1} \mathrm{~F} / \mathrm{cm}^{2}$ or $\sim 10^{-2} \mathrm{~F} / \mathrm{g}$ Au were demonstrated for the button electrodes, and up to $2 \times 10^{-4}$ $\mathrm{F} / \mathrm{cm}^{2}$ or $\sim 5 \mathrm{~F} / \mathrm{g}$ Au for coated electrodes. However, the complex nature of the charge and discharge behaviour of these devices showed that they stored and released energy by more than just double layer capacitance. In particular the oxidation and reduction of gold, and the electrochemical annealing of metastable mesoporous sponges appeared to have contributed significantly to their functionality. Although it is improbable that such devices would have any widespread role in energy storage, their use in capacitive biosensing devices appears promising. 


\section{Acknowledgements}

This work was supported by University of Technology Sydney, the GROW scheme of World Gold Council, and Project AuTek of Mintek, South Africa. The assistance of colleagues at UTS's Microstructural Analysis Unit is also gratefully acknowledged.

\section{References}

1 M. Raney, US Patent 1628190, 1927

2 J.B. Friedrich, D.J. Young, and M.S. Wainwright, J. Electrochem. Soc., 1981, 128, 1845

3 E. Van der Lingen, M.B. Cortie and I. Glaner, South African Patent 2001/5816, 2001

4 M.B. Cortie, E. van der Lingen and G. Pattrick, in Proc. of APNF 2003 Oz Nano 03, Cairns, 19-21st November 2003, World Scientific, Singapore 2004, pp.79-82

5 M. Petri, D.M. Kolb, U. Memmert, H. Meyer, Electrochimica Acta, 2003, 49, 175

6 D.T. Thompson and G.C. Bond, Gold Bulletin, 2000, 33(2), 41

7 G.C. Bond, Gold Bulletin, 2001, 34(4), 117

8 B.E. Conway, V.E. Birss, J. Wojtowicz, J. of Power Sources, 1997, 66, 1

9 D. van Noort, C.F. Mandenius, Biosensors \& Bioelectronics, 2000, 15 203

10 M.J. Natan and B.E. Baker, United States Patent 6,242,264, 2001

11 B. Raguse, W. Yang, G. Stockton, in 28th Annual Condensed Matter and Materials Meeting, 3 - 6 February 2004, Australasian Institute of Physics, Paper WP14

12 A. Toyota, N. Nakashima and T. Sagara, Journal of Electroanalytical Chemistry, 2004, 565335
13 P.N. Bartlett, J.R. Owen, G.S. Attard, J. Elliott, United States Patent 6,503,382, 7th January 2003

14 K. Kamijyou, Metals and Technology (Japan), 1997, 67(1), 38

15 C. Mitterer, H. Lenhart, P.H. Mayrhofer and M. Kathrein, Intermetallics, 2004, 12(5), 579

16 C. Cretu and E. Van der Lingen, Gold Bulletin, 1999, 32, 115

17 R.W. Cahn, Nature, 1998, 396, 523

18 H.B. Liu, J.A. Ascencio, M. Perez-Alvarez, and M.J. Yacaman, Surface Science, 2001, 491, 88

19 K. Dick, T. Dhanasekaran, Z. Xhang, and D. Meisel, J. Am. Chem. Soc, 2002, 124(10), 2312

20 J. Erlebacher, M.J. Aziz, A. Karma, N. Dimitrov and K. Sieradzki, Nature, 2001, 410, 450

21 M.B. Cortie and E. van der Lingen, Materials Forum, 2002, 26, 1

22 S. Strbac and R.R. Adzic, Electrochimica Acta, 1996, 41(18), 2903

23 B.E. Conway, Progress in Surface Science, 1995, 49(4), 331

24 L.D. Burke, A.J. Ahern and A.P. O'Mullane, Gold Bulletin, 2002, 35(1), 3

25 L.D. Burke, Gold Bulletin, 2004, 37, 125

26 C.E. Banks, N.V. Rees and R.G. Compton, J. Phys. Chem. B, 2002, 106, 5810

27 M.A. Schneeweiss and D.M. Kolb, Solid State Ionics, 1997, 94, 171

28 J.G. Velasco. J. Electrochem. Soc., 1997, 144(12), 4142

29 Y. Kim, ECN, February 2002, p.59 (on line at www.ecnmag.com)

30 A. Burke, J. of Power Sources, 2000, 91, 37

31 B.E. Conway and W.G. Pell, J. of Power Sources, 2002, 105, 169

32 C. Berggren and G. Johansson, Anal. Chem., 1997, 69, 3651

33 C.R. Lowe, Current Opinion in Structural Biology, 2000, 10, 428

34 P. Forrer, F. Schlottig, H. Siegenthaler and M. Textor, Journal of Applied Electrochemistry, 2000, 30, 533

35 M.E. Gertner and M. Schlesinger, Electrochemical and Solid-State Letters, 2003, 6(4), 14 